MIDAS

Museus e estudos interdisciplinares

$1 \mid 2013$

Varia

\title{
A museologia como campo de estudo nas universidades portuguesas: esboço de evolução, pertinência e atualidade
}

Museology as a field of study in Portuguese universities: development and relevance

Irene Vaquinhas

\section{OpenEdition}

Journals

Edição electrónica

URL: http://journals.openedition.org/midas/142

DOI: $10.4000 /$ midas. 142

ISSN: $2182-9543$

Editora:

Alice Semedo, Paulo Simões Rodrigues, Pedro Casaleiro, Raquel Henriques da Silva, Ana Carvalho

\section{Refêrencia eletrónica}

Irene Vaquinhas, «A museologia como campo de estudo nas universidades portuguesas: esboço de evolução, pertinência e atualidade », MIDAS [Online], 1 | 2013, posto online no dia 10 abril 2013, consultado no dia 02 maio 2019. URL : http://journals.openedition.org/midas/142 ; DOI : 10.4000/ midas. 142

Este documento foi criado de forma automática no dia 2 Maio 2019

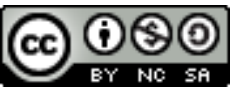

Midas is licensed under a Creative Commons Attribution-NonCommercial-ShareAlike 3.0 International License 


\title{
A museologia como campo de estudo nas universidades portuguesas: esboço de evolução, pertinência e atualidade ${ }^{1}$
}

\author{
Museology as a field of study in Portuguese universities: development and
} relevance

Irene Vaquinhas

\section{Introdução}

1 No ano de 1988, na apresentação do 1.․․ Encontro universitário luso-espanhol sobre a investigação e o ensino na área de museologia, organizado pelo Departamento de Antropologia da Universidade Nova de Lisboa, Henrique Coutinho Gouveia, docente daquela instituição e, ao tempo, diretor do Departamento de Etnologia do Instituto Português do Património Cultural (IPPC), referia que “a presença da museologia no contexto do panorama universitário português é |... pouco significativa sendo escasso o número de instituições em que é ministrado o ensino nessa área", esclarecendo que, nos casos em que essa situação se verifica, “|... reveste |...| um caráter complementar integrando planos de estudo relativos a domínios disciplinares afins” (Gouveia 1991, 1), mais precisamente os curricula de antropologia, de pintura e de escultura (Gil 1991, 33).

2 Apesar do aparente alheamento das universidades portuguesas, nessa área de estudos, tendo os primeiros cursos de pós-graduação sido criados, à margem do ensino oficial público, por organismos estatais, sendo dirigidos a funcionários de museus - caso do curso de "Pós-graduação em Museologia", organizado pelo Instituto Português do Património Cultural (IPPC), em 1981-84 (Freire 1991, 37-48) -, detetavam-se, em seu entender, “|...| sintomas nítidos de mudança", que teriam, a breve trecho, impacto na "multiplicação do 
número de realizações e serviços" e no "incremento de oportunidades profissionais" (Gouveia 1991, 2-3).

3 Aludia-se, em concreto, à criação, ao nível de estudos pós-graduados, do 1.ำ curso de museologia, em Portugal, aberto em 1989, na Universidade Lusíada de Lisboa, e, sobretudo, à "explosão museológica" que então se vivia no país, a qual veio colocar o problema da formação qualificada dos quadros dos museus, tanto do Estado como das múltiplas instituições museais que se encontravam em vias de criação, por iniciativa de municípios, de juntas de freguesias, de empresas privadas e públicas, de associações de defesa do património, entre outras organizações (Correia 1991, 49-51).

4 Em rigor, o número de museus aumentou a partir da década de 1980: se no período anterior ao 25 de Abril de 1974 não chegavam a uma centena, em 1988 existiam, segundo António Nabais, 216 museus, registando-se, no ano de 1993, 260, números estes que, no entanto, ficam aquém de algumas contagens oficiais. Assim, no I Encontro sobre museologia e educação, realizado no Seixal, no ano de 1995, avançou-se o número de 750, o qual é considerado exagerado por diversos especialistas. Já no âmbito do Inquérito aos museus de Portugal, o estudo incide sobre 530 museus, embora, se reconhecesse que, caso tivessem sido adotados critérios mais rigorosos, só $10 \%$ mereciam aquela qualificação (Mendes 2009, 29-30).

5 Contabilidades à parte, o aumento do número de museus, a par das novas funções ou missões que lhes são atribuídas, impulsionou o aparecimento e/ou desenvolvimento de profissões ou de formações capazes de responderem aos desafios lançados à realidade museológica e que, em última instância, se refletiram no ensino superior, criando-se novos programas académicos, contribuindo, assim, para a profissionalização de ocupações ligadas aos museus.

6 Vinte anos depois, no ano de 2009, o panorama do ensino universitário da museologia apresenta-se "irreconhecível", como significativamente o qualificou João Carlos Brigola, na comunicação apresentada no Encontro 0 mercado dos museus e o ensino superior (Brigola 2009, 13-18). Em sua opinião, reforçara-se o investimento humano e material no ensino superior da museologia, tanto ao nível da docência como da diversificação de atividades, o que veio a favorecer a aproximação entre as universidades, os museus e demais instituiç̃oes museais, muito em particular no que respeita à formalização de parcerias em pesquisas e projetos de investigação, bem como na elaboração de catálogos, contratualização de estágios profissionalizantes e publicação de teses.

7 De uma situação de um vazio quase completo transitara-se para um quadro de oferta excessiva, registando-se, no ano de 2009, 9 cursos de mestrado ou de 2. ciclo, número acima das necessidades nacionais em termos de empregabilidade, com a agravante da sua maioria (mais de metade) se concentrar na cidade de Lisboa (Brigola 2009, 14).

Reconhecendo que, na base dessa inflação, se encontram, sobretudo, "factores de ordem orçamental e de ordem profissional que se têm vindo a impor nas direções das universidades", João Carlos Brigola apontava, como soluções para ultrapassar essa situação, a bi-anualidade dos cursos de mestrado ou de 2. ${ }^{\circ}$ ciclo; a criação de 2 ou 3 pólos descentralizados de formação avançada, ligados em rede; o investimento em cursos de $3^{\circ}$ ciclo ou de doutoramento interdepartamentais, assim como a ligação de museus a projetos e a centros de investigação financiados pela Fundação para a Ciência e Tecnologia. 
9 No transato ano letivo de 2010-2011, a formação universitária em museologia, ao nível de cursos de 2..$^{\circ}$ Ciclo ou de mestrado, assegurada por universidades, institutos politécnicos e escolas superiores, regista diferenças relativamente ao quadro traçado para o ano de 2009. O número de cursos aumentou, passando de 9 para 12, contagem que apenas se reporta a todos aqueles que incluem a palavra museologia na sua designação oficial (Quadro I).

Quadro I - Estabelecimentos do ensino superior com cursos de mestrado ou de $2 .^{\circ}$ ciclo na área da museologia (ano letivo 2010-2011)

\begin{tabular}{|c|c|c|}
\hline Instituição & Local & Designação do Mestrado /2. ${ }^{\circ}$ Ciclo \\
\hline $\begin{array}{l}\text { Faculdade de Letras da Universidade de } \\
\text { Lisboa }\end{array}$ & Lisboa & Museologia e Museografia \\
\hline $\begin{array}{l}\text { Faculdade de Ciências Sociais e Humanas } \\
\text { da Univ. Nova de Lisboa }\end{array}$ & Lisboa & Museologia \\
\hline $\begin{array}{l}\text { Instituto Superior de Ciências do } \\
\text { Trabalho e da Empresa }\end{array}$ & Lisboa & Museologia: Conteúdos Expositivos \\
\hline $\begin{array}{l}\text { Faculdade de Belas Artes da } \\
\text { Universidade de Lisboa }\end{array}$ & Lisboa & Museologia e Museografia \\
\hline Escola Superior de Artes Decorativas & Lisboa & $\begin{array}{l}\begin{array}{l}\text { Museografia e Gestão em Artes } \\
\text { Decorativas }\end{array} \\
\text { a }\end{array}$ \\
\hline Universidade Lusíada & Lisboa & Museologia \\
\hline $\begin{array}{l}\text { Universidade Lusófona de Humanidades } \\
\text { e Tecnologias }\end{array}$ & Lisboa & Museologia \\
\hline $\begin{array}{l}\text { Faculdade de Letras da Universidade do } \\
\text { Porto }\end{array}$ & Porto & $\begin{array}{l}\text { Museologia, } \quad \text { Especialização } \quad \text { em } \\
\text { Museologia }\end{array}$ \\
\hline $\begin{array}{l}\text { Faculdade de Belas Artes da } \\
\text { Universidade do Porto }\end{array}$ & Porto & $\begin{array}{l}\text { Est. Artísticos, Especialização Estudos } \\
\text { Museológicos e Curatoriais }\end{array}$ \\
\hline $\begin{array}{l}\text { Faculdade de Letras da Universidade de } \\
\text { Coimbra }\end{array}$ & Coimbra & História, Especialização em Museologia \\
\hline Universidade de Évora & Évora & Museologia \\
\hline $\begin{array}{l}\text { Univ. Açores (Departamento de História, } \\
\text { Filosofia e Ciências Sociais) }\end{array}$ & $\begin{array}{l}\text { Ponta } \\
\text { Delgada }\end{array}$ & $\begin{array}{l}\text { Património, } \quad \text { Museologia } \\
\text { Desenvolvimento }\end{array}$ \\
\hline
\end{tabular}

Fonte: sites web

10 O seu número seria superior, se se contabilizassem os cursos em áreas afins (ciências do património, estudos curadoriais ou educação museal, entre outros), de 1. e 2. ciclo que oferecem cadeiras de museologia nos seus programas curriculares, bem como as pós- 
graduações (não conferentes de grau) ou as formações feitas em museus e que se destinam prioritariamente aos seus profissionais ${ }^{2}$ (Quadros II e III).

Quadro II - Alguns estabelecimentos do ensino superior com cursos de mestrado ou de $2 .^{\circ}$ ciclo em áreas conexas à museologia (listagem não completa)

\begin{tabular}{|c|c|c|c|}
\hline Instituição & Nível & Local & Designação do Mestrado /2. ${ }^{\circ}$ Ciclo \\
\hline Universidade do Algarve & 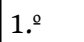 & Faro & Património Cultural (Art., Hist. e Arqueol.) \\
\hline Universidade Lusíada & 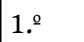 & Lisboa & Ciências do Património \\
\hline FCSH da UNL & 2.. & Lisboa & $\begin{array}{l}\text { Conservação e Restauro; História e } \\
\text { Património da Ciência, Tecnologia e } \\
\text { Inovação }\end{array}$ \\
\hline FCSH da UNL & $2 . \stackrel{\circ}{0}$ & Lisboa & Ciências da Conservação \\
\hline $\begin{array}{l}\text { Fac. de Belas Artes da Univ. de } \\
\text { Lisboa e Fundação C. Gulbenkian }\end{array}$ & 2..$^{\circ}$ & Lisboa & Estudos Curatoriais \\
\hline $\begin{array}{l}\text { Instituto Superior Técnico da } \\
\text { Universidade Técnica }\end{array}$ & 2. .9 & Lisboa & $\begin{array}{l}\text { Pós-Graduação em Recuperação e } \\
\text { Conservação do Património Construído }\end{array}$ \\
\hline Universidade Aberta & 2.. & Lisboa & Mestrado em Estudos do Património \\
\hline Universidade Lusíada & 2.. & Lisboa & $\begin{array}{l}\text { Educação e Promoção Cultural pelo } \\
\text { Património }\end{array}$ \\
\hline Universidade de Coimbra & 2.. & Coimbra & Conservação e Restauro \\
\hline Universidade de Évora & 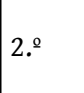 & Évora & $\begin{array}{l}\text { Gestão e Valorização do Património } \\
\text { Histórico e Cultural }\end{array}$ \\
\hline $\begin{array}{l}\text { Escola Superior de Educação do } \\
\text { Porto }\end{array}$ & 2.. & Porto & Estudos de Gestão do Património \\
\hline $\begin{array}{l}\text { Escola Superior de Tecnologia de } \\
\text { Tomar }\end{array}$ & $2 . .9$ & Tomar & Mestrado em Conservação e Restauro \\
\hline
\end{tabular}

\section{FONTE: SITES WEB}

Quadro III - Estabelecimentos do ensino superior com cursos de pós-graduação na área da museologia

\begin{tabular}{|l|l|l|}
\hline Instituição & Local & Designação da Pós-graduação \\
\hline Escola Superior de Tecnologia de Tomar & Tomar & Gestão de Museus e Colecções de Arqueologia \\
\hline
\end{tabular}

FONTE: SITES WEB 
11 Trata-se de um número excessivo de cursos, em confronto com outros países. Mesmo adotando a seleção com base no numerus clausus são todos anuais, exceto o 2. ciclo em "História, especialização em Museologia", da Faculdade de Letras da Universidade de Coimbra, que pratica a bi-anualidade desde a sua 1. edição, no ano letivo de 1998-1999.

Segundo dados internacionais, considerados não exaustivos, no ano de 1998-1999, em França, a formação académica, no domínio da museologia, repartia-se por 1 curso do 1. ․․ ciclo, 8 de 2.ำ ciclo e 7 do 3. ciclo $^{3}$; em Espanha e nos Estados-Unidos, em igual período de tempo, a oferta, no 1. caso, nas áreas de museologia e de gestão cultural e patrimonial, alcançava os 18 cursos, enquanto, no 2.. caso, se polarizava por 126 cursos de $2 .^{\circ}$ Ciclo e 10 de 3.. Ciclo (Laporte, Tolosana 2001, 95-97; Landry 2001, 99-108).

Quadro IV - Estabelecimentos do ensino superior com cursos de $2 .^{\circ}$ e $3 .^{\circ}$ ciclos na área da museologia (Ano de 1998-1999)

\begin{tabular}{|l|l|l|l|}
\hline Países & 2.o Ciclo & 3.o Ciclo & \\
\hline & & & \\
\hline França & 8 & 7 & \\
\hline Espanha & & & $18^{*}$ \\
\hline Estados-Unidos da América & 126 & 10 & \\
\hline & & & \\
\hline *em gestão cultural, gestão do património e museologia & & & \\
\hline
\end{tabular}

FONTE: ALLARD, LEFEBVRE 2001, 32; 95-108.

13 A análise mais pormenorizada do caso português permite concluir pelo reforço de cursos em Lisboa, em relação ao ano de 2009, divididos entre a Faculdade de Letras, a Faculdade de Ciências Sociais e Humanas da Universidade Nova, o ISCTE, a Faculdade de Belas Artes, a Escola Superior de Artes Decorativas, as Universidades Lusíada e Lusófona, mantendose, sem alteração, a localização das formações académicas fora da capital (em Coimbra, em Évora, no Porto e em Ponta Delgada) (Quadro V).

Quadro V - Distribuição geográfica de estabelecimentos com cursos de mestrado ou de $2 .{ }^{\circ}$ ciclo em museologia

\begin{tabular}{|l|l|l|}
\hline Localidade & N.. & $\%$ \\
\hline Lisboa & 7 & 58.3 \\
\hline Porto & 2 & 16.7 \\
\hline Coimbra & 1 & 8.3 \\
\hline Évora & 1 & 8.3 \\
\hline
\end{tabular}




\begin{tabular}{|l|l|l|}
\hline Ponta Delgada & 1 & 8.3 \\
\hline Total & 12 & 100 \\
\hline
\end{tabular}

14 A estratégia de desenvolvimento local passa, no entanto, em alguns casos pela extensão da docência em museologia, fora da respetiva área geográfica, situação que se aplica à Universidade de Évora que apoia a formação neste campo de estudos na Universidade de Cabo Verde ${ }^{4}$. Ou, pelo contrário, como ocorre na Universidade Lusófona, o curso socorrese de um leque alargado de professores brasileiros, no âmbito de parcerias assinadas com instituições de ensino superior do Brasil.

A instauração de um novo patamar de excelência com a criação de cursos de $3^{\circ}$ ciclo ou de doutoramento foi uma das respostas universitárias à proliferação de formações na área da museologia com consequências na sua relativa desvalorização, bem como à procura social de qualificações académicas mais prestigiantes, procurando-se assim assegurar uma preparação para funções de direção ou para quadros superiores em instituições museais, num meio profissional muito sujeito às vicissitudes políticas ou às iniciativas locais.

No ano letivo de 2010-2011, foram em número de três, as instituições de ensino superior que disponibilizaram esse tipo de cursos: a Faculdade de Letras da Universidade do Porto em parceria com a Faculdade de Belas Artes desta cidade, o Centro de Estudos de História e Filosofia da Ciência da Universidade de Évora e a Universidade Lusófona (Quadro VI). Já o curso de 3. ${ }^{\circ}$ Ciclo da Faculdade de Ciências Sociais e Humanas da Universidade Nova de Lisboa se enquadra na área da História da Arte sendo designado por "História da Arte, especialidade Museologia e Património Artístico".

Quadro VI - Universidades com cursos de doutoramento ou de $3 .^{\circ}$ ciclo em museologia

\begin{tabular}{|c|c|c|}
\hline Instituição & Local & Designação do Doutoramento /3. ${ }^{\circ}$ Ciclo \\
\hline $\begin{array}{l}\text { Faculdade de Letras/Faculdade de Belas } \\
\text { Artes da Universidade do Porto }\end{array}$ & Porto & 3. ${ }^{\circ}$ Ciclo em Museologia \\
\hline $\begin{array}{l}\text { Universidade de Évora (Centro de Estudos } \\
\text { de História e Filosofia da Ciência) }\end{array}$ & Évora & $\begin{array}{l}\text { História e Filosofia da Ciência - com } \\
\text { especialidade Museologia }\end{array}$ \\
\hline Universidade Lusófona & Lisboa & Museologia \\
\hline FCSH da Universidade Nova de Lisboa & Lisboa & $\begin{array}{l}\text { História da Arte, especialidade } \\
\text { Museologia e Património Artístico }\end{array}$ \\
\hline
\end{tabular}

17 Embora, o espaço temporal objeto de análise seja demasiado curto para permitir conclusões definitivas, os dados parecem sugerir que, neste nível de qualificação, o panorama se inverte relativamente à oferta de cursos de 2.. ciclo, esbatendo-se a importância da cidade de Lisboa relativamente a outros pólos de formação especializada.

18 Esta conclusão afigura-se poder ser corroborada por outro tipo de informações. Se tomarmos como base de pesquisa, a listagem das bolsas de doutoramento, concedidas pela Fundação para a Ciência e Tecnologia (FCT), de 2007 a 2010, sobre temas de museologia, 
esta demonstra, no que respeita às instituições conferentes de grau, a dispersão universitária.

19 No que concerne, pois, ao período de 2007-2010, num total de 11 doutoramentos em curso, correspondendo a 2,7\%, da totalidade de projetos inscritos no ramo de "História e Arqueologia" (em número de 407), estes polarizam-se por instituições do ensino superior, com práticas científicas há muito consolidadas (Quadro VII).

Quadro VII - Bolsas de doutoramento na área da museologia concedidas pela FCT de acordo com a Instituição que confere o grau (2007-2010)

\begin{tabular}{|l|l|l|}
\hline N.o & $\%$ & Instituições \\
\hline 2 & 18,2 & FLUP - Universidade do Porto \\
\hline 2 & 18,2 & FLUC - Universidade de Coimbra \\
\hline 2 & 18,2 & UE - Universidade de Évora \\
\hline 2 & 18,2 & UL - Faculdade Ciências de Lisboa \\
\hline 1 & 9,1 & FCSH da Univ. Nova de Lisboa \\
\hline 1 & 9,1 & Universidade Complutense de Madrid \\
\hline 1 & 9,1 & Universidade Lusófona de Lisboa \\
\hline & & \\
\hline 11 & 100 & \\
\hline
\end{tabular}

FONTE: SERVIÇO DE BOLSAS DA FCT, DFRH BOLSEIROS DA ÁREA DA HISTÓRIA E ARQUEOLOgIA

A falta de informações estatísticas impede conhecer a evolução numérica dos efetivos estudantis, inscritos e diplomados, nos $2 .^{\circ}$ e $3 .^{\circ}$ ciclos, de forma a ter-se uma ideia, mesmo aproximada, da produção da mão-de-obra potencial para museus. Apenas em dois casos (Faculdade de Letras da Universidade de Coimbra e Universidade de Évora) se dispõe de dados sobre o número de diplomados, os quais apontam para uma média anual baixa: 4,7 e 2,6, respetivamente (Quadro VIII).

Quadro VIII - Alunos diplomados / teses defendidas na área de museologia de 2001 a 2011 (FLUC e Universidade de Évora)

\begin{tabular}{|l|l|l|}
\hline Anos letivos & FLUC & U. Évora \\
\hline $2001-2002$ & 2 & 0 \\
\hline $2002-2003$ & 12 & 2 \\
\hline $2003-2004$ & 0 & 4 \\
\hline $2004-2005$ & 2 & 5 \\
\hline
\end{tabular}




\begin{tabular}{|l|l|l|}
\hline $2005-2006$ & 3 & 5 \\
\hline $2006-2007$ & 2 & 1 \\
\hline $2007-2008$ & 6 & 2 \\
\hline $2008-2009$ & 5 & 1 \\
\hline $2009-2010$ & 9 & 6 \\
\hline $2010-2011$ & 6 & \\
\hline Total & 47 & 26 \\
\hline Média anual & 4.7 & 2.6 \\
\hline
\end{tabular}

FONTE: SERVIÇO ACADÉMICOS DA UNIVERSIDADE DE ÉVORA /DIVISÃo DE ESTUdOS PÓS-GRADUADOS / ESTATÍSTICAS PARA O MCTES; ${ }^{\circ}$ DE TESES/RELATÓRIOS DEFENDIDOS NA FLUC

21 Tenha-se em linha de conta que, no caso da FLUC, o curso de museologia é bi-anual, ou seja, só tem lugar de dois em dois anos.

De modo idêntico, pouco se sabe sobre a inserção dos novos mestres no mercado de trabalho dos museus ou em setores culturais afins.

\section{Análise dos planos curriculares}

23 A resposta universitária às mudanças ocorridas no campo das atividades museais não se circunscreve apenas à abertura de novos cursos, à elevação do nível de estudos e ao investimento na captação de novos públicos.

A análise dos planos curriculares dos $2^{\circ}$ ciclos permite detetar uma evolução que visa acompanhar as mudanças estruturais ocorridas no mundo dos museus, mais precisamente a passagem de políticas museais assentes na transmissão e na conservação patrimonial para modelos empresariais orientados para uma adequada gestão económica e financeira, a qual implica a valorização, junto do público, dos respetivos patrimónios.

Nesse sentido, os planos demonstram uma vertente profissionalizante centrada em quatro eixos principais:

- em 1. lugar, na comunicação e na receção de saberes, sobretudo ao nível dos discursos expositivos, das práticas e das tecnologias da informação ou inclusivamente dos estudos de público. Por outras palavras: a importância atribuída na atualidade às estatísticas de frequência de museus, bem como os cortes nas dotações orçamentais obrigam os museus a prestarem grande atenção tanto aos públicos como aos não-públicos, na designação de Pierre Bourdieu e de Alain Darbel, preocupações que se afiguram estar presentes nos programas curriculares de museologia.

- em 2. lugar, um vetor de disciplinas focalizadas na problematização teórica da museologia, em grande parte escorada no conhecimento histórico e outras áreas científicas próximas (antropologia, sociologia...);

- em 3. lugar, uma incidência disciplinar na gestão e na administração de museus (planeamento, modelos de gestão e de financiamento...), dando-se relevância ao estudo de 
estratégias de marketing que visam, em última instância, atrair aos museus grupos subrepresentados;

- em 4. lugar, um núcleo de disciplinas centradas nas questões patrimoniais e na história da arte (tratamento, conservação, enquadramento jurídico, restauro, entre outros) ${ }^{5}$.

Quadro IX - Áreas das disciplinas dos planos curriculares de museologia (por ordem descendente de incidência temática)

\begin{tabular}{|l|l|l|}
\hline Áreas de especialização & N.o & $\%$ \\
\hline Comunicação & 19 & 20.9 \\
\hline História / Teoria Museológica & 18 & 19.7 \\
\hline Conservação / Restauro & 10 & 10.9 \\
\hline Investigação & 9 & 9.8 \\
\hline Gestão & 8 & 8.79 \\
\hline Património & 7 & 7.69 \\
\hline História / História da Arte & 7 & 7.69 \\
\hline Arquitectura & 6 & 6.6 \\
\hline Museografia & 3 & 3.29 \\
\hline Gestão / Programação & 3 & 3.29 \\
\hline Sem identificação & 1 & 1.09 \\
\hline Total & 91 & 100 \\
\hline
\end{tabular}

Por outras palavras, os planos curriculares evidenciam uma aproximação aos campos das indústrias culturais, aos métodos da gestão e às políticas educativas, procurando, desta forma, o ensino superior ir ao encontro das funções desempenhadas pelos museus da atualidade. Pode aplicar-se, neste contexto, uma afirmação proferida por Dubois, em 1998, para quem "O desenvolvimento da autonomia estatutária, financeira e pedagógica da universidade obriga a considerá-la como uma instituição cujas missões são definidas pelo Estado, mas igualmente como um conjunto de profissões científicas e, cada vez mais, como uma empresa produzindo conhecimentos e diplomados para um mercado" (Cordier 2001, 25).

Os curricula não deixam, contudo, de manifestar alguns paradoxos. No momento em que as instituições museais atribuem grande importância às questões educativas, estas ocupam, nos planos analisados, um papel secundário, sendo enquadradas por outras matérias. De igual forma, está subalternizada a investigação científica, para a qual o ensino superior está diretamente vocacionado, e que importa incentivar. A principal exceção respeita sobretudo aos museus de ciências, os quais têm uma longa tradição neste campo, ocupando os estudos de coleç̃os um espaço privilegiado nas suas atividades, em 
estreita ligação com o ensino universitário. A formação em história da arte tem também uma prática regular de visitas a museus e de estudos das obras artísticas.

Convém lembrar que, em Portugal, as cadeiras de Museologia começaram por se alojar na Licenciatura de Antropologia (caso da Faculdade de Ciências Sociais e Humanas da Universidade Nova de Lisboa), a partir dos anos 1980, onde constituía uma "introdução à museologia, com particular incidência no caso dos museus de etnologia, de modo a revelar aos alunos um campo de conhecimento e potencialmente uma via de profissionalização" (Gouveia 1991, 225).

Trata-se de uma situação distinta da vizinha Espanha, país onde a museologia começou por se incorporar nas Faculdades de Belas Artes, bem como de Filosofia e Letras, mais precisamente, neste segundo caso, na Licenciatura em História (situação que se aplica, entre outras, às Universidades Complutense, de Madrid, e à de Salamanca).

Muitos outros aspetos poderiam ainda ser explorados a partir da relação entre museus e ensino superior, como é o caso, entre outras hipóteses possíveis, do enquadramento profissional do corpo docente, no sentido de determinar o peso de técnicos superiores de museus no ensino lecionado, bem como a internacionalização dos cursos e o seu impacto no exterior captados através da frequência de alunos estrangeiros, entre outros aspetos.

Convém, ainda, realçar que esta área da docência tem estado muita atenta às potencialidades das novas tecnologias e enveredado por novos caminhos de difusão e de partilha de saberes, dinamizando redes sociais na internet, como a Museologia.porto, a qual foi iniciada por Alice Semedo, docente da Faculdade de Letras da Universidade do Porto; o blog No mundo dos museus ou a lista Museum, plataforma de difusão de informação no âmbito da museologia e do património cultural. Esta última nasceu no contexto do Mestrado em "Museologia e Património Cultural" da Faculdade de Letras da Universidade de Coimbra, fruto do empenho e da dedicação de um dos seus docentes, José d 'Encarnação, tendo sido publicamente reconhecida a sua utilidade social, ao ser-lhe atribuído o prémio APOM 2010 da "melhor comunicação on-line".

\section{Em conclusão}

Face à antiguidade dos museus, a museologia, como campo de estudos no ensino universitário, é muito recente, encontrando-se ainda em processo de construção e de reconhecimento. Porém, independentemente das mudanças em curso, o levantamento efetuado, para o ano letivo de 2010-2011, a partir dos sites web da grande maioria dos estabelecimentos de ensino superior portugueses, demonstra, de uma forma inequívoca, que a museologia não só entrou em força nas universidades portuguesas como constitui a matriz de uma profunda renovação da formação profissional dos museus e demais instituições museais.

33 Ao assegurar novas competências e saberes tem contribuído para alargar o leque de atividades disponibilizadas pelos museus em áreas tão distintas como a dinamização sócio-cultural, a educação, a conservação, a exposição, etc. A análise dos programas curriculares também deixou claro que se tende a articular a vertente interdisciplinar com a preocupação em englobar as diversas funções dos museus, associando-se questões de ordem teórica com aspetos práticos e técnicos.

Porém, no momento em que existem doze cursos de formação pós-graduada em Portugal, importa, acima de tudo, refletir no ensino que é ministrado, avaliar das suas vantagens e 
insuficiências, e incentivar uma colaboração mais estreita com os museus. Impõe-se neste campo determinar, com maior precisão, que formação se pretende dar: se um museólogo generalista, se um professional especializado, se um "educador de museu". Aliás, esta última categoria é defendida por numerosos autores (Mendes 2009, 41-43).

No nosso país, por oposição a outros onde a oferta editorial é extensíssima, não existem livros-guia ou manuais, adequados às exigências de cursos especializados e tendo como casos de estudo museus portugueses. Não deixa de ser um pouco paradoxal, face à ausência de publicações desta natureza (um exemplo de todos conhecido é a obra coordenada por Maria Beatriz Rocha-Trindade, Iniciação à museologia (Lisboa, Universidade Aberta, 1993), a qual constituiu, ao tempo, um trabalho pioneiro, que se conheça com mais pormenor, museus espanhóis ou britânicos, do que os museus portugueses ou que estes sejam dados como casos exemplificativos.

Creio que se impõe que a docência universitária se projete também neste campo, o que pode ajudar a constituir também uma forma de autoavaliação, no sentido de se alcançar um melhor conhecimento do que se faz em museologia, como se faz e para quem se faz. Impõe-se também, e creio que as universidades também poderiam ajudar bastante nesse campo, avaliar do impacto dos museus nas comunidades em que se inserem. As potencialidades do ensino superior da museologia constituem, pois, um campo em aberto.

\section{BIBLIOGRAFIA}

Actas do $1 .$. encontro universitário luso-espanhol sobre a investigação e o ensino na área de museologia, Ethnologia, n.․ 6, Julho/Dezembro 1991, Departamento de Antropologia, Faculdade de Ciências Sociais da Universidade Nova de Lisboa.

Alarcão, Adília. 1998. “Museus e universidades que relação?”. Jornal de Coimbra, 2 a 8 de Dezembro, ano XII, n.ำ 584 .

Lefebvre, Bernard, e Michel Allard, dir. 1994. Le musée un projet éducatif, Montréal (Québec): Les Éditions Logiques.

Carreño, Francisco Javier Zubiaur. 2004. Curso de Museologia. Gijón: Ediciones Tréa, S. L.

Cordier, Jean-Pierre. 2001. "Les formations muséales en France". In La formation en muséologie et en éducation muséale à travers le monde, ed. Michel Allard, e Bernard Lefebvre, 15-50. Québec: Editions Multimondes.

Brigola, João Carlos, 2009. “O actual ensino universitário da Museologia - uma reflexão crítica e uma proposta”. Museologia.pt (3):13-18.

Correia, Joaquim de Oliveira. 1991. "O projecto de licenciatura em História da Arte na U.A.L. e o ensino da Museologia", Actas do 1.ำencontro universitário luso-espanhol sobre a investigação e o ensino na área de museologia, Ethnologia, n.․ 6, Julho/Dezembro 1991, Departamento de Antropologia, Faculdade de Ciências Sociais da Universidade Nova de Lisboa: 49-51.

Encarnação, José d'. 1991. "Perspectivas para um curso de museologia na Faculdade de Letras de Coimbra", Actas do 1.encontro universitário luso-espanhol sobre a investigação e o ensino na área de 
museologia, Ethnologia, n. 6, Julho/Dezembro 1991, Departamento de Antropologia, Faculdade de Ciências Sociais da Universidade Nova de Lisboa: 69-74.

Freire, Fernando António Baptista. 1991. "Problemática da formação museológica em Portugal: o curso de museologia do IPPC (1981-1984)”, Actas do 1.encontro universitário luso-espanhol sobre a investigação e o ensino na área de museologia, Ethnologia, n. 6, Julho/Dezembro 1991, Departamento de Antropologia, Faculdade de Ciências Sociais da Universidade Nova de Lisboa: 37-48.

Fundação para a Ciência e Tecnologia (FCT) - DFRH Bolseiros da área da História e Arqueologia.

Gil, Fernando Bragança. 1991. "Ensino da museologia: a situação em Portugal", Actas do 1.ำ encontro universitário luso-espanhol sobre a investigação e o ensino na área de museologia, Ethnologia, n.. 6, Julho/Dezembro 1991, Departamento de Antropologia, Faculdade de Ciências Sociais da Universidade Nova de Lisboa: 23-35.

Gouveia, Henrique Coutinho. 1991. "Algumas considerações introdutórias”, "Museologia e antropologia ensino e investigação no Departamento de Antropologia da Universidade Nova de Lisboa", Actas do 1.encontro universitário luso-espanhol sobre a investigação e o ensino na área de museologia, Ethnologia, n. 6, Julho/Dezembro 1991, Departamento de Antropologia, Faculdade de Ciências Sociais da Universidade Nova de Lisboa: 1-5; 53-65.

Hernandez Hernandez, Francisca. 1991. "Panorama actual de la museologia como disciplina universitaria", Actas do 1.ำencontro universitário luso-espanhol sobre a investigação e o ensino na área de museologia, Ethnologia, n.․ 6, Julho/Dezembro 1991, Departamento de Antropologia, Faculdade de Ciências Sociais da Universidade Nova de Lisboa: 121-131.

Landry, Anik. 2001. "Les programmes de formation en muséologie dans les institutions supérieures d' enseignement aux États-Unis”. In La formation en muséologie et en éducation muséale à travers le monde, ed. Michel Allard, e Bernard Lefebvre, 99-108. Québec: Editions Multimondes.

Lapa, Sofia. 2009. "Estudo de museus: projectos universitários", Newsletter. Associação Portuguesa de Historiadores da Arte, Dezembro 2008: 8.

Laporte, Antoni, e Montse L. Tolosana. 2001. "Les études de muséologie en Espagne”. In La formation en muséologie et en éducation muséale à travers le monde, ed. Michel Allard, e Bernard Lefebvre, 95-97. Québec: Editions Multimondes.

Mayrand, Pierre, e Mário C. Moutinho. 2007. “Le musée local de la nouvelle generation au Portugal, un pas en avant dans la gestion communautaire qualitative: essai d'interprétation épistemologique." Cadernos de Museologia (28):45-55.

Mendes, José Maria Amado. 2009. “O papel educativo dos museus: evolução histórica e tendências actuais". In "Educação e museus: novas correntes", Estudos do Património. Museus e Educação, 29-47; 155-165. Coimbra: Imprensa da Universidade, Estudos. Humanidades.

“Museus em Rede". 2010. Boletim da Rede Portuguesa de Museus, (36):5.

Nabais, António José C. Maia. 1993. “Museus na actualidade”. In Iniciação à Museologia, coord. Maria Beatriz Rocha-Trindade, 63-76. Lisboa: Universidade Aberta.

\section{ANEXOS}

Webgrafia

http://museologiaporto.ning.com/ (consultado em março 19, 2011). 
http://www.educaedu.com.pt/mestrado-em-museologia-e-museografiamestrado-21771.html (consultado em março 18, 2011).

http://www.educaedu.com.pt/mestrado-em-estudos-curatoriais-mestrado-21774.html (consultado em março 18, 2011).

http://www.educaedu.com.pt/mestrado-em-museologia-mestrado-23613.html (consultado em março 18, 2011).

http://www.educaedu.com.pt/mestrado-em-conservacao-e-restauromestrado-20413.html (consultado em março 18, 2011).

http://www.educaedu.com.pt/mestrado-em-ciencias-da-conservacaomestrado-20416.html (consultado em março 18, 2011).

http://www.educaedu.com.pt/mestrado-em-museologia-conteudos-expositivosmestrado-16831.html (consultado em março 18, 2011).

http://www.educaedu.com.pt/pos-graduacao-em-recuperacao-e-conservacao-dopatrimonio-construido-pos-graduacao-22311.html (consultado em março 18, 2011).

http://www.educaedu.com.pt/mestrado-em-museologia-mestrado-17717.html (consultado em março 18, 2011).

http://www.educaedu.com.pt/mestrado-em-museologia-mestrado-17717.html (consultado em março 18, 2011).

http://www.educaedu.com.pt/mestrado-em-conservacao-e-restauromestrado-22801.html (consultado em março 18, 2011).

http://www.educaedu.com.pt/mestrado-em-museologia-mestrado-23310.html (consultado em março 18, 2011).

http://www.educaedu.com.pt/mestrado-em-museologia-mestrado-18503.html (consultado em março 18, 2011).

http://www.educaedu.com.pt/mestrado-em-educacao-e-promocao-cultural-pelopatrimonio-mestrado-23756.html (consultado em março 18, 2011).

http://www.educaedu.com.pt/licenciatura-em-ciencias-do-patrimoniolicenciatura-23651.html (consultado em março 18, 2011).

http://www.educaedu.com.pt/mestrado-em-estudos-do-patrimoniomestradoo-20502.htmlform-info (consultado em março 18, 2011).

www.ese.ipp.pt/cursos/mestrados/ (consultado em março 27, 2011).

http://portal.ipt.pt/portal/portal/mestradoConserRest (31-03-2011; 22.12).

www.ese.ipp.pt/cursos/mestrados/( consultado em março 27, 2011).

www.estt.ipt.pt (consultado em março 31, 2011).

www.dhfcs.uac.pt (consultado em março 20, 2011).

www.ese.ipp (consultado em março 27, 2011).

mpac.fba.up.pt/blog (consultado em março 20, 2011).

http://www.fba.ul.pt/portal/page?_pageid=401,1016045\&dad=portal\&_schema=PORTAL (consultado em março 21, 2011). 
http://xa.ying.com/kg/groups/1518869/289286451/name/programa_doutoramento.pdf (consultado em março 30, 2011).

http://www.ulusofona.pt/index.php/faculdade-de-arquitectura-urbanismo-geografia-eartes/doutoramento/museologia (consultado em março 30, 2011).

http://sigarra.up.pt/flup/planos_estudos_geral,formview?p_Pe_1575 (consultado em março 30, 2011).

http://www.fress.pt/Default.aspx?Tag=CONTENT\&Contentld=68 (consultado em março 21, 2011).

http://sigarra.up.flup/planos_estudos_geral.formview?p_Pe=1175 (consultado em março $21,2011)$.

www.uevora.pt (consultado em abril 9, 2011).

http://ler.letras.up.pt/site/default.aspx?qry=id03id1319id2320\&sum=sim

www.uc.pt/fluc/noticias/museum_premio_apom/ (consultado em abril 9, 2011).

www.fenix.iscte.pt (consultado em abril 7, 2011).

www.unicv.edu.cv/...de...cabo/index.php? (consultado em março 31, 2011)

Anexo I

\begin{tabular}{|l|l|}
\hline \multicolumn{2}{|l|}{ Listagem dos Estabelecimentos de Ensino Superior consultados } \\
\hline 1 & Academia Militar \\
\hline 2 & Escola Superior de Enfermagem de Coimbra \\
\hline 3 & Escola Superior de Enfermagem de Lisboa \\
\hline 4 & Escola Superior de Enfermagem do Porto \\
\hline 5 & Escola Superior de Hotelaria e Turismo do Estoril \\
\hline 6 & Escola Superior Náutica Infante D. Henrique \\
\hline 7 & $\begin{array}{l}\text { Instituto Politécnico da Guarda - Escola Superior de Educação, Comunicação e Desporto da } \\
\text { Guarda }\end{array}$ \\
\hline 8 & Instituto Politécnico da Guarda - Escola Superior de Saúde da Guarda \\
\hline 9 & Instituto Politécnico da Guarda - Escola Superior de Tecnologia e Gestão da Guarda \\
\hline 10 & Instituto Politécnico da Guarda - Escola Superior de Turismo e Hotelaria \\
\hline 11 & Instituto Politécnico de Beja - Escola Superior Agrária de Beja \\
\hline 12 & Instituto Politécnico de Beja - Escola Superior de Educação de Beja \\
\hline 13 & Instituto Politécnico de Bragança - Escola Superior Agrária de Bragança \\
\hline
\end{tabular}




\begin{tabular}{|c|c|}
\hline 14 & $\begin{array}{l}\text { Instituto Politécnico de Bragança - Escola Superior de Comunicação, Administração e } \\
\text { Turismo de Mirandela }\end{array}$ \\
\hline 15 & Instituto Politécnico de Bragança - Escola Superior de Educação de Bragança \\
\hline 16 & Instituto Politécnico de Bragança - Escola Superior de Tecnologia e de Gestão de Bragança \\
\hline 17 & $\begin{array}{l}\text { Instituto Politécnico de Castelo Branco - Escola Superior de Artes Aplicadas de Castelo } \\
\text { Branco }\end{array}$ \\
\hline 18 & Instituto Politécnico de Castelo Branco - Escola Superior de Educação de Castelo Branco \\
\hline 19 & Instituto Politécnico de Castelo Branco - Escola Superior de Gestão de Idanha-a-Nova \\
\hline 20 & Instituto Politécnico de Castelo Branco - Escola Superior de Tecnologia de Castelo Branco \\
\hline 21 & Instituto Politécnico de Coimbra - Escola Superior Agrária de Coimbra \\
\hline 22 & Instituto Politécnico de Coimbra - Escola Superior de Educação de Coimbra \\
\hline 23 & Instituto Politécnico de Coimbra - Escola Superior de Tecnologia da Saúde de Coimbra \\
\hline 24 & $\begin{array}{l}\text { Instituto Politécnico de Coimbra - Escola Superior de Tecnologia e Gestão de Oliveira do } \\
\text { Hospital }\end{array}$ \\
\hline 25 & $\begin{array}{l}\text { Instituto Politécnico de Coimbra - Instituto Superior de Contabilidade e Administração de } \\
\text { Coimbra }\end{array}$ \\
\hline 26 & Instituto Politécnico de Coimbra - Instituto Superior de Engenharia de Coimbra \\
\hline 27 & Instituto Politécnico de Leiria - Escola Superior de Artes e Design das Caldas da Rainha \\
\hline 28 & Instituto Politécnico de Leiria - Escola Superior de Educação e Ciências Sociais de Leiria \\
\hline 29 & Instituto Politécnico de Leiria - Escola Superior de Saúde de Leiria \\
\hline 30 & Instituto Politécnico de Leiria - Escola Superior de Tecnologia e Gestão de Leiria \\
\hline 31 & $\begin{array}{l}\text { Instituto Politécnico de Leiria - Escola Superior de Turismo e Tecnologia do Mar de } \\
\text { Peniche }\end{array}$ \\
\hline 32 & Instituto Politécnico de Lisboa - Escola Superior de Comunicação Social de Lisboa \\
\hline 33 & Instituto Politécnico de Lisboa - Escola Superior de Educação de Lisboa \\
\hline 34 & Instituto Politécnico de Lisboa - Escola Superior de Música de Lisboa \\
\hline 35 & Instituto Politécnico de Lisboa - Escola Superior de Teatro e Cinema de Lisboa \\
\hline 36 & Instituto Politécnico de Lisboa - Escola Superior de Teatro e Cinema de Lisboa \\
\hline 37 & Instituto Politécnico de Lisboa - Escola Superior de Tecnologia da Saúde de Lisboa \\
\hline
\end{tabular}




\begin{tabular}{|c|c|}
\hline 38 & $\begin{array}{l}\text { Instituto Politécnico de Lisboa - Instituto Superior de Contabilidade e Administração de } \\
\text { Lisboa }\end{array}$ \\
\hline 39 & Instituto Politécnico de Lisboa - Instituto Superior de Engenharia de Lisboa \\
\hline 40 & Instituto Politécnico de Portalegre - Escola Superior Agrária de Elvas \\
\hline 41 & Instituto Politécnico de Portalegre - Escola Superior da Saúde de Portalegre \\
\hline 42 & Instituto Politécnico de Portalegre - Escola Superior de Tecnologia e Gestão de Portalegre \\
\hline 43 & Instituto Politécnico de Santarém - Escola Superior Agrária de Santarém \\
\hline 44 & Instituto Politécnico de Santarém - Escola Superior de Desporto de Rio Maior \\
\hline 45 & Instituto Politécnico de Santarém - Escola Superior de Educação de Santarém \\
\hline 46 & Instituto Politécnico de Santarém - Escola Superior de Gestão e Tecnologia de Santarém \\
\hline 47 & Instituto Politécnico de Santarém - Escola Superior de Saúde de Santarém \\
\hline 48 & Instituto Politécnico de Setúbal - Escola Superior de Ciências Empresariais de Setúbal \\
\hline 49 & Instituto Politécnico de Setúbal - Escola Superior de Educação de Setúbal \\
\hline 50 & Instituto Politécnico de Setúbal - Escola Superior de Tecnologia de Setúbal \\
\hline 51 & Instituto Politécnico de Setúbal - Escola Superior de Tecnologia do Barreiro \\
\hline 52 & Instituto Politécnico de Tomar - Escola Superior de Gestão de Tomar \\
\hline 53 & Instituto Politécnico de Tomar - Escola Superior de Tecnologia de Abrantes \\
\hline 54 & Instituto Politécnico de Tomar - Escola Superior de Tecnologia de Tomar \\
\hline 55 & Instituto Politécnico de Viana do Castelo - Escola Superior Agrária de Ponte de Lima \\
\hline 56 & Instituto Politécnico de Viana do Castelo - Escola Superior de Educação de Viana do Castelo \\
\hline 57 & Instituto Politécnico de Viana do Castelo - Escola Superior de Saúde de Viana do Castelo \\
\hline 58 & $\begin{array}{l}\text { Instituto Politécnico de Viana do Castelo - Escola Superior de Tecnologia e Gestão de Viana } \\
\text { do Castelo }\end{array}$ \\
\hline 59 & Instituto Politécnico de Viseu - Escola Superior Agrária de Viseu \\
\hline 60 & Instituto Politécnico de Viseu - Escola Superior de Educação de Viseu \\
\hline 61 & Instituto Politécnico de Viseu - Escola Superior de Saúde de Viseu \\
\hline 62 & Instituto Politécnico de Viseu - Escola Superior de Tecnologia e Gestão de Viseu \\
\hline 63 & Instituto Politécnico do Cávado e do Ave - Escola Superior de Gestão \\
\hline
\end{tabular}




\begin{tabular}{|c|c|}
\hline 64 & Instituto Politécnico do Cávado e do Ave - Escola Superior de Tecnologia \\
\hline 65 & Instituto Politécnico do Porto - Escola Superior de Educação do Porto \\
\hline 66 & Instituto Politécnico do Porto - Escola Superior de Estudos Industriais e de Gestão \\
\hline 67 & Instituto Politécnico do Porto - Escola Superior de Música e Artes do Espectáculo \\
\hline 68 & Instituto Politécnico do Porto - Escola Superior de Tecnologia da Saúde do Porto \\
\hline 69 & Instituto Politécnico do Porto - Escola Superior de Tecnologia e Gestão de Felgueiras \\
\hline 70 & $\begin{array}{l}\text { Instituto Politécnico do Porto - Instituto Superior de Contabilidade e Administração do } \\
\text { Porto }\end{array}$ \\
\hline 71 & Instituto Politécnico do Porto - Instituto Superior de Engenharia do Porto \\
\hline 72 & Instituto Superior de Ciências Policiais e Segurança Interna \\
\hline 73 & ISCTE - Instituto Universitário de Lisboa \\
\hline 74 & Universidade Aberta \\
\hline 75 & Universidade da Beira Interior \\
\hline 76 & Universidade da Madeira \\
\hline 77 & Universidade da Madeira - Escola Superior de Enfermagem da Madeira \\
\hline 78 & Universidade de Aveiro \\
\hline 79 & Universidade de Aveiro - Instituto Superior de Contabilidade e Administração de Aveiro \\
\hline 80 & Universidade de Coimbra - Colégio de Artes \\
\hline 81 & Universidade de Coimbra - Faculdade de Ciências do Desporto e Educação Física \\
\hline 82 & Universidade de Coimbra - Faculdade de Ciências e Tecnologia \\
\hline 83 & Universidade de Coimbra - Faculdade de Direito \\
\hline 84 & Universidade de Coimbra - Faculdade de Economia \\
\hline 85 & Universidade de Coimbra - Faculdade de Farmácia \\
\hline 86 & Universidade de Coimbra - Faculdade de Letras \\
\hline 87 & Universidade de Coimbra - Faculdade de Medicina \\
\hline 88 & Universidade de Coimbra - Faculdade de Psicologia e de Ciências da Educação \\
\hline 89 & Universidade de Évora \\
\hline
\end{tabular}




\begin{tabular}{|c|c|}
\hline 90 & Universidade de Évora - Escola de Artes \\
\hline 91 & Universidade de Évora - Escola de Ciências e Tecnologia \\
\hline 92 & Universidade de Évora - Escola de Ciências Sociais \\
\hline 93 & Universidade de Évora - Escola Superior de Enfermagem de São João de Deus \\
\hline 94 & Universidade de Évora - Instituto de Investigação e Formação Avançada \\
\hline 95 & Universidade de Lisboa - Faculdade de Belas-Artes \\
\hline 96 & Universidade de Lisboa - Faculdade de Ciências \\
\hline 97 & Universidade de Lisboa - Faculdade de Direito \\
\hline 98 & Universidade de Lisboa - Faculdade de Farmácia \\
\hline 99 & Universidade de Lisboa - Faculdade de Letras \\
\hline 100 & Universidade de Lisboa - Faculdade de Medicina \\
\hline 101 & Universidade de Lisboa - Faculdade de Medicina Dentária \\
\hline 102 & Universidade de Lisboa - Faculdade de Psicologia \\
\hline 103 & Universidade de Lisboa - Instituto de Ciências Sociais \\
\hline 104 & Universidade de Lisboa - Instituto de Educação \\
\hline 105 & Universidade de Lisboa - Instituto de Geografia e Ordenamento do Território \\
\hline 106 & Universidade de Trás-os-Montes e Alto Douro \\
\hline 107 & Universidade de Trás-os-Montes e Alto Douro - Escola de Ciências da Vida e do Ambiente \\
\hline 108 & Universidade de Trás-os-Montes e Alto Douro - Escola de Ciências e Tecnologia \\
\hline 109 & Universidade de Trás-os-Montes e Alto Douro - Escola de Ciências Humanas e Sociais \\
\hline 110 & Universidade do Algarve - Escola Superior de Educação e Comunicação \\
\hline 111 & Universidade do Algarve - Escola Superior de Gestão, Hotelaria e Turismo de Faro \\
\hline 112 & Universidade do Algarve - Escola Superior de Saúde de Faro \\
\hline 113 & Universidade do Algarve - Faculdade de Ciências Humanas e Sociais \\
\hline 114 & Universidade do Algarve - Faculdade de Economia \\
\hline 115 & Universidade do Algarve - Instituto Superior de Engenharia \\
\hline 116 & Universidade do Minho - Escola de Ciências \\
\hline
\end{tabular}




\begin{tabular}{|c|c|}
\hline 117 & Universidade do Minho - Instituto de Ciências Sociais \\
\hline 118 & Universidade do Minho - Instituto de Educação \\
\hline 119 & Universidade do Minho - Instituto de Letras e Ciências Humanas \\
\hline 120 & Universidade do Porto - Faculdade de Arquitectura \\
\hline 121 & Universidade do Porto - Faculdade de Belas-Artes \\
\hline 122 & Universidade do Porto - Faculdade de Ciências \\
\hline 123 & Universidade do Porto - Faculdade de Ciências da Nutrição e da Alimentação \\
\hline 124 & Universidade do Porto - Faculdade de Desporto \\
\hline 125 & Universidade do Porto - Faculdade de Direito \\
\hline 126 & Universidade do Porto - Faculdade de Economia \\
\hline 127 & Universidade do Porto - Faculdade de Engenharia \\
\hline 128 & Universidade do Porto - Faculdade de Farmácia \\
\hline 129 & Universidade do Porto - Faculdade de Letras \\
\hline 130 & Universidade do Porto - Faculdade de Medicina \\
\hline 131 & Universidade do Porto - Faculdade de Medicina \\
\hline 132 & Universidade do Porto - Faculdade de Medicina Dentária \\
\hline 133 & Universidade do Porto - Faculdade de Psicologia e de Ciências da Educação \\
\hline 134 & Universidade do Porto - Instituto de Ciências Biomédicas de Abel Salazar \\
\hline 135 & Universidade dos Açores \\
\hline 136 & Universidade Nova de Lisboa - Escola Nacional de Saúde Pública \\
\hline 137 & Universidade Nova de Lisboa - Faculdade de Ciências e Tecnologia \\
\hline 138 & Universidade Nova de Lisboa - Faculdade de Ciências Médicas \\
\hline 139 & Universidade Nova de Lisboa - Faculdade de Ciências Sociais e Humanas \\
\hline 140 & Universidade Nova de Lisboa - Faculdade de Direito \\
\hline 141 & Universidade Nova de Lisboa - Faculdade de Economia \\
\hline 142 & Universidade Nova de Lisboa - Instituto de Higiene e Medicina Tropical \\
\hline 143 & Universidade Nova de Lisboa - Instituto de Tecnologia Química e Biológica \\
\hline
\end{tabular}




\begin{tabular}{|l|l|}
\hline 144 & Universidade Nova de Lisboa - Instituto Superior de Estatística e Gestão de Informação \\
\hline 145 & Universidade Técnica de Lisboa - Faculdade de Arquitectura \\
\hline 146 & Universidade Técnica de Lisboa - Faculdade de Medicina Veterinária \\
\hline 147 & Universidade Técnica de Lisboa - Faculdade de Motricidade Humana \\
\hline 148 & Universidade Técnica de Lisboa - Instituto Superior de Agronomia \\
\hline 149 & Universidade Técnica de Lisboa - Instituto Superior de Ciências Sociais e Políticas \\
\hline 150 & Universidade Técnica de Lisboa - Instituto Superior de Economia e Gestão \\
\hline 151 & Universidade Técnica de Lisboa - Instituto Superior Técnico \\
\hline 152 & Universidade Lusíada de Lisboa \\
\hline 153 & Faculdade de Belas Artes da Universidade de Lisboa \\
\hline 154 & Faculdade de Belas Artes da Universidade do Porto \\
\hline 135 & Escola Superior de Artes Decorativas de Lisboa \\
\hline 156 & Universidade Lusófona de Humanidades e Tecnologias de Lisboa \\
\hline 157 & Universidade Católica Portuguesa \\
\hline 158 & Universidade de Évora \\
\hline
\end{tabular}

\section{Anexo II}

Disciplinas curriculares dos cursos de mestrado e de $2^{\circ}$ ciclo de museologia em estabelecimentos do ensino superior (2010-2011)

\begin{tabular}{|l|l|}
\hline Título & Estabelecimentos \\
\hline Museologia e Museografia & \\
\hline Comunicação Visual & U. Lisboa \\
\hline Programação de Museus e de Exposições & U. Lisboa \\
\hline Colecções e Espaços Museológicos & U. Lisboa \\
\hline Operações Museográficas & U. Lisboa \\
\hline Princípios da Gestão de Museus e Legislação do Património & U. Lisboa \\
\hline & \\
\hline Museologia & FCSH da UN Lisboa \\
\hline
\end{tabular}




\begin{tabular}{|c|c|}
\hline Arquitectura e Administração de Museus & FCSH da UN Lisboa \\
\hline Conservação Preventiva & FCSH da UN Lisboa \\
\hline Economia, Gestão e Direito do Património & FCSH da UN Lisboa \\
\hline Exposição, Investigação e Comunicação & FCSH da UN Lisboa \\
\hline História e Teoria da Museologia & FCSH da UN Lisboa \\
\hline Programação Museológica & FCSH da UN Lisboa \\
\hline Sistemas Documentação & FCSH da UN Lisboa \\
\hline Museologia: Conteúdos Expositivos & ISCTE \\
\hline Questões de Museologia & ISCTE \\
\hline A Nação e a sua Construção Cultural & ISCTE \\
\hline Conservação e Manutenção & ISCTE \\
\hline Públicos, Consumo e Lazer & ISCTE \\
\hline Museus e Novas Tecnologias & ISCTE \\
\hline Estudo de Colecções & ISCTE \\
\hline Museus e História Contemporânea & ISCTE \\
\hline Design Expositivo & ISCTE \\
\hline Políticas Museológicas & ISCTE \\
\hline Antropologia Visual & ISCTE \\
\hline Gestão e Intervenção Cultural & ISCTE \\
\hline Contextos Museológicos & ISCTE \\
\hline Arquitectura de Museus & ISCTE \\
\hline Museologia e Museografia & F Belas Artes da U Lisboa \\
\hline & F Belas Artes da U Lisboa \\
\hline Teoria e História da Museologia & F Belas Artes da U Lisboa \\
\hline Praxis e Gestão Museológica & F Belas Artes da U Lisboa \\
\hline
\end{tabular}




\begin{tabular}{|c|c|}
\hline Shadow Curating & F Belas Artes da U Lisboa \\
\hline Design de Apresentação (Equipamento) & F Belas Artes da U Lisboa \\
\hline Conservação Preventiva e Teoria do Restauro & F Belas Artes da U Lisboa \\
\hline Programação de Museus e Exposições & F Belas Artes da U Lisboa \\
\hline Projecto; Estratégia, Metodologia , Práticas e Implementação & F Belas Artes da U Lisboa \\
\hline Design de Apresentação II (Comunicação) & F Belas Artes da U Lisboa \\
\hline Museografia e Gestão em Artes Decorativas & ESArtes Decorativas Lisboa \\
\hline Arquitectura & ESArtes Decorativas Lisboa \\
\hline Ciências Sociais e Humanas & ESArtes Decorativas Lisboa \\
\hline Conservação & ESArtes Decorativas Lisboa \\
\hline Museografia & ESArtes Decorativas Lisboa \\
\hline 2o Ciclo em História, Especialização em Museologia & FLUC \\
\hline Museus e História & FLUC \\
\hline Cultura Material e Museologia & FLUC \\
\hline Património Cultural & FLUC \\
\hline Discurso Museológico & FLUC \\
\hline Gestão Museológica & FLUC \\
\hline Museus, Investigação e Educação & FLUC \\
\hline Museologia & Univ. Lusíada Lisboa \\
\hline Património Museológico & Univ. Lusíada Lisboa \\
\hline Museologia & Univ. Lusíada Lisboa \\
\hline Planeamento, Organização e Gestão dos Museus & Univ. Lusíada Lisboa \\
\hline Conservação e Restauro do Objecto Museológico & Univ. Lusíada Lisboa \\
\hline Função Social dos Museus & Univ. Lusíada Lisboa \\
\hline
\end{tabular}




\begin{tabular}{|c|c|}
\hline Conservação Preventiva & Univ. Lusíada Lisboa \\
\hline Organização e Gestão de Colecções & Univ. Lusíada Lisboa \\
\hline Metodologia do Trabalho Científico & Univ. Lusíada Lisboa \\
\hline Museologia & Univ. Lusófona Lisboa \\
\hline A Função Social dos Museus & Univ. Lusófona Lisboa \\
\hline Museologia e Património & Univ. Lusófona Lisboa \\
\hline Museologia e Pensamento Contemporâneo & Univ. Lusófona Lisboa \\
\hline Estudos Aprofundados em Museologia & Univ. Lusófona Lisboa \\
\hline Museografia & Univ. Lusófona Lisboa \\
\hline Museologia e Desenvolvimento Cultural & Univ. Lusófona Lisboa \\
\hline Metodologias de Investigação em Museologia & Univ. Lusófona Lisboa \\
\hline Museologia Contemporânea. Práticas e Conceitos & Univ. Lusófona Lisboa \\
\hline Museologia & Univ. Évora \\
\hline Teoria e História da Museologia & Univ. Évora \\
\hline História Social da Arte e da Cultura & Univ. Évora \\
\hline Museus e Arquitectura & Univ. Évora \\
\hline Património, Museu e Território & Univ. Évora \\
\hline Gestão de Museus & Univ. Évora \\
\hline Incorporação e Documentação & Univ. Évora \\
\hline Processos Expositivos & Univ. Évora \\
\hline Museologia - Especialização em Museologia & FLUP \\
\hline Arquitectura de Museus & FLUP \\
\hline Estudos e Gestão de Colecções & FLUP \\
\hline Museus e Centros de Arte Contemporânea & FLUP \\
\hline
\end{tabular}




\begin{tabular}{|c|c|}
\hline Museus e Museologia & FLUP \\
\hline Riscos, Museus e Vulnerabilidades & FLUP \\
\hline Conservação Preventiva & FLUP \\
\hline Museus, Identidades e Representações & FLUP \\
\hline Organização e Gestão de Museus & FLUP \\
\hline Política e Práticas de Comunicações em Museus & FLUP \\
\hline Projecto, Espaço e Comunicação em Museus & FLUP \\
\hline Tecnologias da Informação e Comunicação em Museus & FLUP \\
\hline Estudos Artísticos - Espec. Estudos Museológicos e Curadoriais & F Belas Artes da U Porto \\
\hline A Exposição: Teorias e Práticas I & F Belas Artes da U Porto \\
\hline Conceitos de Museologia Contemporânea & F Belas Artes da U Porto \\
\hline Estudos de Arte Contemporânea & F Belas Artes da U Porto \\
\hline A Exposição: Teorias e Práticas II & F Belas Artes da U Porto \\
\hline Conservação da Arte Contemporânea & F Belas Artes da U Porto \\
\hline Crítica Contemporânea da Arte & F Belas Artes da U. Porto \\
\hline Património, Museologia e Desenvolvimento & U. Açores \\
\hline Museologia e Antropologia Cultural & U. Açores \\
\hline Conservação e Restauro & U. Açores \\
\hline História da Arte e do Património & U. Açores \\
\hline Património, Identidade e Desenvolvimento & U. Açores \\
\hline Cidade e Património Urbano & U. Açores \\
\hline Gestão e Direito do Património & U. Açores \\
\hline Museologia e Património Cultural & U. Açores \\
\hline Sociologia da Cultura e dos Públicos & U. Açores \\
\hline
\end{tabular}




\section{NOTAS}

1. O texto deste artigo reproduz, com ligeiras alterações, a comunicação oral apresentada no Seminário Redes Regionais de Museus, realizado em Coimbra, por iniciativa da Direção Regional da Cultura do Centro, a 13 de abril de 2011. A pesquisa que conduziu a este estudo realizou-se em março e abril de 2011 e incidiu num total de cerca de 160 instituições de ensino superior, entre universidades, Institutos Politécnicos e Escolas Superiores (ANEXO I). Agradeço, muito reconhecida, à Sra. Dra. Olga Martinho, da Fundação para a Ciência e a Tecnologia (Ministério da Educação e Ciência, Portugal), bem como ao Sr. Prof. Doutor Filipe Themudo Barata, da Universidade de Évora, as informações que gentilmente me forneceram para a elaboração deste trabalho.

2. As ações de formação promovidas por museus da Rede Portuguesa de Museus são, de uma forma geral, elencadas no Boletim da Rede Portuguesa de Museus. A título exemplificativo veja-se, entre outros, Museus em Rede (2010).

3. Em rigor, são em número de 50, os cursos na área patrimonial, lecionados em 30 estabelecimentos de ensino e 18 cidades universitárias (Cordier 2001, 18; 32).

4. Mais precisamente no Departamento de Ciências Sociais e Humanas da Universidade de Cabo Verde, na cidade da Praia (ilha de Santiago). Está em curso "o projeto de análise e diagnóstico do panorama patrimonial e museológico dos municípios caboverdeanos", cfr. www.unicv.edu.cv/... de...cabo/index.php? (consultado em março 31, 2011).

5. Os planos curriculares dos diversos cursos de mestrado ou de $2 .$. ciclo na área de museologia, disponíveis no ano letivo de 2010-2011, nas instituições do ensino superior portuguesas, encontram-se elencados no Anexo II.

\section{RESUMOS}

Nesta comunicação é feito o levantamento da formação académica em museologia que é oferecida pelas instituições de ensino superior em Portugal (universidades, institutos politécnicos e escolas superiores), procurando-se igualmente captar, através da análise das estruturas curriculares dos vários cursos de 2.e 3. Ciclos, como é que o meio universitário tem reagido ao dinamismo observado nas funções e atividades atuais desempenhadas pelos museus, bem como avaliar do seu contributo para a profissionalização do respetivo pessoal técnico.

This paper surveys the academic training provided by higher education establishments in Portugal (universities, polytechnics and further education colleges) in the field of museology. By analysing the curricula of the various courses offered at Master's and Doctorate level, it gauges their response to the dynamism currently observable in the activities and functions of museums, and assesses their contribution to the professional training of specialised personnel in this domain. 


\section{ÍNDICE}

Keywords: higher education, master's and doctorate levels, museum, Portugal, history of museological training

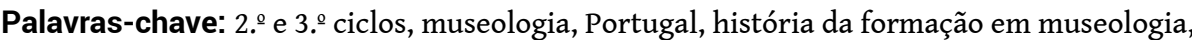
museus

\section{AUTOR}

\section{IRENE VAQUINHAS}

Professora catedrática da Faculdade de Letras da Universidade de Coimbra. É diretora do 3. Ciclo em Altos Estudos em História; diretora do 2.․ Ciclo em História, Especialização em Museologia desde 2006 e membro do Centro de História da Sociedade e da Cultura da Universidade de Coimbra (FCT). Publicou, entre outros, os seguintes trabalhos: Violência, justiça e sociedade rural. Os campos de Coimbra, Montemor-o-Velho e Penacova de 1858 a 1918, Porto, Afrontamento, 1996; "Senhoras e mulheres" na sociedade portuguesa do século XIX, Lisboa, Edições Colibri, 2000; Nome de Código 33856. Os "jogos de fortuna ou azar" em Portugal entre a repressão e a tolerância (De finais do século XIX a 1927), Lisboa, Livros Horizonte, 2006. Colaborou na História de Portugal, Direção de José Mattoso, V vol. - O Liberalismo (1807-1890), Lisboa, Círculo de Leitores, 1993. Coordenou o vol. III da obra História da Vida Privada em Portugal, (A Época Contemporânea), dirigida por José Mattoso, sendo também autora de diversos capítulos (Lisboa, Círculo de Leitores, 2011). Tem efetuado cursos e seminários em universidades estrangeiras. irenemcv@fl.uc.pt 